\title{
The primordial density perturbation in the curvaton scenario
}

\author{
David H. Lyth ${ }^{1}$, Carlo Ungarelli ${ }^{2}$ and David Wands ${ }^{2}$ \\ (1) Department of Physics, Lancaster University, Lancaster LA1 4YB, U. K. \\ (2) Institute of Cosmology and Gravitation, University of Portsmouth, Portsmouth PO1 2EG, U. K.
} (February 2, 2008)

\begin{abstract}
We analyse the primordial density perturbation when it is generated by a 'curvaton' field different from the inflaton. In some cases this perturbation may have large isocurvature components, fully correlated or anti-correlated with the adiabatic component. It may also have a significant nonGaussian component. All of these effects are calculated in a form which will enable direct comparison with current and forthcoming observational data.
\end{abstract}

PACS numbers: $98.80 . \mathrm{Cq}$

Preprint PU-ICG-02/15, astro-ph/0208055v2

\section{INTRODUCTION}

It is now clear that the origin of structure in the Universe is a primordial density perturbation, existing already when cosmological scales start to enter the horizon. Observation is consistent with the hypothesis that the density perturbation is perfectly adiabatic, Gaussian and scale-independent, but significant departures from this state of affairs is still allowed by the data. In particular it is not excluded that the adiabatic density perturbation may be accompanied by a significant isocurvature density perturbation [1] 2].

Inflation provides a natural origin for the perturbation, since it converts the vacuum fluctuation of each light free scalar field into a classical scale-independent perturbation. One or more of these field perturbations may cause the primordial density perturbation.

It is usually assumed that inflation involves a slowlyrolling field, dubbed the inflaton, whose value determines the end of inflation. The perturbation in the inflaton field cannot cause an isocurvature perturbation, but does inevitably cause at some level an adiabatic perturbation. The usual hypothesis [3] is that the inflaton is solely responsible for the observed adiabatic density perturbation. Under this 'inflaton hypothesis' significant nonGaussianity is excluded in the usual one-field models 幽. In multi-field models, where there is a family of possible inflaton trajectories curved in field space, significant non-Gaussianity is possible [6,7] but apparently only at the expense of extreme fine-tuning of the initial condition that specifies the inflaton trajectory. Any isocurvature density perturbation must be caused by the perturbation of some non-inflaton field. Under the inflaton hypothesis this means that the isocurvature density perturbation (if present) depends on different physical parameters from the adiabatic density perturbation. As a result, an isocurvature perturbation of observable magnitude would require fine-tuning of the physical parameters, or else some as-yet unforseen connection between them.

An alternative hypothesis [8,9.10,11 is that the adiabatic density perturbation originates from the pertur- bation in some 'curvaton' field different from the inflaton. In this scenario the adiabatic density perturbation is generated only after inflation, from an initial condition which cgrresponds to a purely isocurvature perturbation [12]. The object of the present paper is to explore the nature of the primordial density perturbation under this hypothesis. In the curvaton scenario, significant non-Gaussianity may easily be present because the curvaton density is proportional to the square of the curvaton field. Also, the curvaton density perturbation can lead, after curvaton decay, to isocurvature perturbations in the densities of the various components of the cosmic fluid. These, which we term 'residual' isocurvature components, are either fully correlated or fully anti-correlated with the adiabatic density perturbation, with a calculable and generally significant relative magnitude.

The paper is organized as follows. We deal in Section II with the adiabatic perturbation and its possible nonGaussianity. In Section III we formulate the description of isocurvature perturbations, in a way which will allow us to analyse CDM, baryon and neutrino perturbations in a unified manner. In Section IV we calculate the residual isocurvature perturbations of cold dark matter (CDM) and baryons. In Section $\mathrm{V}$ we give a general formalism for describing the primordial neutrino isocurvature perturbation, taking into account for the first time the crucial issue of lepton number. Then we use it to calculate the residual isocurvature neutrino perturbation. Our conclusions are summarised in Section VI.

\footnotetext{
${ }^{*}$ This conversion mechanism has been considered also in the pre big bang scenario [13:14. In this scenario though, the required scale-invariant curvaton field perturbations will be generated only if the curvaton has a non-trivial coupling and for particular initial conditions $[15,16]$.
} 


\section{THE CURVATURE PERTURBATION}

\section{A. The curvature perturbation and the primordial density perturbations}

From the viewpoint of observation the 'primordial' epoch is the one a few Hubble times before the smallest cosmological scale approaches the horizon. Taking that scale to enclose say $10^{6}$ solar masses, the primordial epoch corresponds to temperature of order $10 \mathrm{keV}$ which is after nucleosynthesis. Leaving aside the possibility of a particle decaying after nucleosynthesis, the content of the Universe at the primordial epoch is therefore rather well known. There are photons, practically massless neutrinos, baryons and (assuming it is already in existence) cold dark matter, with the radiation dominating the energy density. The corresponding primordial density perturbation has an adiabatic mode

$$
\frac{1}{4} \frac{\delta \rho_{\gamma}}{\rho_{\gamma}}=\frac{1}{4} \frac{\delta \rho_{\nu}}{\rho_{\nu}}=\frac{1}{3} \frac{\delta \rho_{B}}{\rho_{B}}=\frac{1}{3} \frac{\delta \rho_{\mathrm{cdm}}}{\rho_{\mathrm{cdm}}},
$$

which leaves the local ratio of number densities unperturbed. Non-adiabatic or isocurvature modes are defined by

$$
\begin{aligned}
\mathcal{S}_{B} & \equiv \frac{\delta \rho_{B}}{\rho_{B}}-\frac{3}{4} \frac{\delta \rho_{\gamma}}{\rho_{\gamma}} \\
\mathcal{S}_{\mathrm{cdm}} & \equiv \frac{\delta \rho_{\mathrm{cdm}}}{\rho_{\mathrm{cdm}}}-\frac{3}{4} \frac{\delta \rho_{\gamma}}{\rho_{\gamma}} \\
\mathcal{S}_{\nu} & \equiv \frac{3}{4} \frac{\delta \rho_{\nu}}{\rho_{\nu}}-\frac{3}{4} \frac{\delta \rho_{\gamma}}{\rho_{\gamma}} .
\end{aligned}
$$

In this section we focus on the adiabatic mode, which is known to be the dominant one responsible for structure formation, and return to the possible isocurvature modes in the next section.

The primordial adiabatic density perturbation is associated with a spatial curvature perturbation. Following [17,18 we define the curvature perturbation $\zeta$ on spatial slices of uniform density $\rho$ with the line element

$$
\mathrm{d} \ell^{2}=a^{2}(1+2 \zeta) \delta_{i j} \mathrm{~d} x^{i} \mathrm{~d} x^{j} .
$$

The quantity $\zeta$ is related to the density perturbation, $\delta \rho$, and curvature perturbation, $\psi$, on a generic slicing (using the sign convention of Ref. [19]) by the gauge-invariant formulat

$$
\begin{aligned}
\zeta & =-\psi-H \Delta t \\
& =-\psi-H \frac{\delta \rho}{\dot{\rho}} .
\end{aligned}
$$

\footnotetext{
${ }^{\dagger}$ In general a gauge corresponds to a definite slicing and threading of spacetime, but in this paper only the former is relevant so that 'gauge-invariant' can be taken to mean 'independent of the slicing'.
}

where $\Delta t$ is the displacement of the generic slicing from the uniform-density slicing. On super-horizon scales $\zeta$ is practically identical with the curvature $\mathcal{R}$ defined on slices orthogonal to comoving worldlines. The quantity $\zeta \approx \mathcal{R}$ is useful on such scales because it is timeindependent [20,21,22, 18, 3] provided that the pressure perturbation is adiabatic, meaning that $\delta P=c_{s}^{2} \delta \rho$, where the adiabatic sound speed $c_{s}^{2}=\dot{P} / \dot{\rho}$. This is guaranteed if there exists a universal equation of state $P(\rho)$. If the pressure perturbation is not adiabatic, then $\zeta \approx \mathcal{R}$ changes according to the equation 20,21.23]

$$
\dot{\zeta}=-\frac{H}{\rho+P} \delta P_{\text {nad }},
$$

where $\delta P_{\text {nad }}=\delta P-c_{s}^{2} \delta \rho$.

In the conventional inflaton model for the origin of structure purely adiabatic perturbations are generated due to quantum fluctuations in the single scalar field driving inflation. Thus the curvature perturbation, $\zeta_{*}$, calculated shortly after Hubble-exit $(k=a H)$ determines the curvature perturbation until that scale re-enters the Hubble scale during the subsequent radiation or matterdominated era. By contrast, we are interested in a scenario where the curvature perturbation generated on large-scales during inflation in the very early universe is negligible.

In the rest of this section we describe the generation of the large-scale curvature perturbation, $\zeta$, in the curvaton scenario, amplifying the outline given in the original paper [9] (see also [24]). We deal with the simplest version of the curvaton scenario, where the curvature perturbation is caused exclusively by a single 'curvaton' field distinct from the inflaton field.

\section{B. Generating the curvaton field perturbation}

During inflation the Hubble parameter $H$ is assumed to be slowly varying, $\epsilon_{H} \equiv \dot{H} / H^{2} \ll 1$. The inflaton (if it exists) is supposed to produce a negligible curvature perturbation. Demanding that it is (say) less than $1 \%$ of the observed value implies $[3] V_{*}^{\frac{1}{4}}<2 \times 10^{15} \mathrm{GeV}$, which in turn implies [D] that the primordial gravitational waves will have no detectable effect on the CMB anisotropy. Conversely, the detection of an effect would rule out the curvaton hypothesis (an "anti-smoking gun") 25,24].

The curvaton field $\sigma$ is supposed to be practically free during inflation, with small effective mass $\left(\left|V_{\sigma \sigma}\right| \ll H^{2}\right.$ where a subscript $\sigma$ denotes $\partial / \partial \sigma)$. It follows that on super-horizon scales there is a Gaussian perturbation with an approximately scale-independent spectrum given by

$$
\mathcal{P}_{\delta \sigma}^{\frac{1}{2}}(k) \approx \frac{H_{*}}{2 \pi},
$$

where the ${ }^{*}$ denotes the epoch of horizon exit $k=a H$. (The normalization of the spectrum [3] is such that $\mathcal{P}_{\delta \sigma}^{\frac{1}{2}}$ 
specifies the typical magnitude of a spatial fluctuation in $\delta \sigma$ on a physical scale $a / k$.) The spectral index specifying the slight scale-dependence is given by [9,25]

$$
n-1 \equiv \mathrm{d} \ln \left(\mathcal{P}_{\delta \sigma}\right) / \mathrm{d} \ln k=2 \eta_{\sigma \sigma}-2 \epsilon_{H},
$$

where $\eta_{\sigma \sigma} \equiv V_{\sigma \sigma} / 3 H^{2}$.

After the smallest cosmological scale leaves the horizon, the curvature perturbation remains negligible until after the curvaton starts to oscillate. As a result, the curvaton field evolves in unperturbed spacetime. To follow the evolution it is assumed that the curvaton field has no significant coupling to other fields or, to be more precise, that the effect of any coupling can be integrated out to give a possibly time-dependent effective potential $V$. With these assumptions, the curvaton field smoothed on the smallest cosmological scale evolves along each comoving worldline according to the unperturbed field equation

$$
\ddot{\sigma}+3 H \dot{\sigma}+V_{\sigma}=0 .
$$

Making the first-order approximation $\delta\left(V_{\sigma}(\mathbf{x}, t)\right) \approx$ $V_{\sigma \sigma}(t) \delta \sigma(\mathbf{x}, t)$, its inhomogeneous perturbation satisfies

$$
\ddot{\delta \sigma}+3 H \dot{\delta \sigma}+V_{\sigma \sigma} \delta \sigma=0 .
$$

Assuming only that the evolution of $\delta \sigma$ is linear, the Gaussianity and (slight) scale-dependence of the original quantity are preserved. The fractional perturbation $\delta \sigma(\mathbf{x}) / \sigma$ does not evolve at all if the potential is either sufficiently flat (so that $\sigma$ and $\delta \sigma$ are both constant) or quadratic (so that $\sigma$ and $\delta \sigma$ satisfy the same equation).

The field remains over-damped until the Hubble parameter falls below the curvaton mass $m_{\sigma}$. The curvaton field will then start to oscillate about its vacuum value (taken to be $\sigma=0$ ) with an amplitude which decreases with time. Even if the potential of the curvaton field is not quadratic, after a few Hubble times we can make the approximation $V \approx \frac{1}{2} m_{\sigma}^{2} \sigma^{2}$. The fractional field perturbation then has some constant value,

$$
\left(\frac{\delta \sigma}{\sigma}\right)=q\left(\frac{\delta \sigma}{\sigma}\right)_{*}
$$

where the factor $q$ is time-independent because the oscillation is around a quadratic minimum of the potential. In particular, if the effective potential for the curvaton is quadratic (or sufficiently flat) throughout its evolution, $q=1$. Using Eq. (9), the spectrum of the fractional field perturbation at this stage is

$$
\mathcal{P}_{\delta \sigma / \sigma}^{\frac{1}{2}}=\frac{q}{2 \pi} \frac{H_{*}}{\sigma_{*}} .
$$

The energy density in the oscillating field is

$$
\rho_{\sigma}(\mathbf{x}, t)=m_{\sigma}^{2} \tilde{\sigma}^{2}(\mathbf{x}, t),
$$

where $\tilde{\sigma}(\mathbf{x}, t)$ is the amplitude of the oscillation. The perturbation in $\rho_{\sigma}$ depends on the curvaton field perturbation through both a linear and a quadratic term.
Assuming, for the moment, that the linear term dominates (which we shall see is demanded by the data) we have

$$
\frac{\delta \rho_{\sigma}}{\rho_{\sigma}} \approx 2 \frac{\delta \sigma}{\sigma}=2 q\left(\frac{\delta \sigma}{\sigma}\right)_{*} .
$$

We shall return to consider the possible contribution from the quadratic term in section IID.

\section{Generating the curvature perturbation}

So far we have reached the epoch just after the Hubble parameter falls below the curvaton mass, and the curvaton oscillation starts. At this stage, it is supposed that the dominant energy density comes from radiation. The curvaton, though, is supposed to be fairly long-lived, while decaying comfortably before nucleosynthesis. So long as the decay-rate is negligible $(\Gamma \ll H)$, we have $\rho_{\sigma} \propto a^{-3}$ and $\rho_{r} \propto a^{-4}$, leading to $\rho_{\sigma} / \rho_{r} \propto a$. It is this increase in the relative curvaton energy density which generates the curvature perturbation.

To analyse the generation of the curvature perturbation (and also, later on, to discuss possible isocurvature perturbations produced by the curvaton decay), it is convenient to consider the curvature perturbations $\zeta_{i}$ corresponding to the separate components of the energy density. These are defined on slices of uniform $\rho_{i}$, corresponding to the gauge-invariant definition

$$
\zeta_{i} \equiv-\psi-H\left(\frac{\delta \rho_{i}}{\dot{\rho}_{i}}\right) .
$$

In particular, evaluating $\zeta_{\sigma}$ for the curvaton on unperturbed $(\psi=0)$ hypersurfaces when the curvaton starts to oscillate, we have

$$
\zeta_{\sigma}=\frac{1}{3} \frac{\delta \rho_{\sigma}}{\rho_{\sigma}}=\frac{2}{3} q\left(\frac{\delta \sigma}{\sigma}\right)_{*} .
$$

The total curvature perturbation (7) can then be written as the weighted sum

$$
\zeta=(1-f) \zeta_{r}+f \zeta_{\sigma}
$$

where the relative contribution of the curvaton to the total curvature is given by

$$
f=\frac{3 \rho_{\sigma}}{4 \rho_{r}+3 \rho_{\sigma}} .
$$

Thus the curvaton perturbation, $\zeta_{\sigma}$, can initially be described as an isocurvature perturbation since $f \rightarrow 0$, i.e., $\rho_{\sigma} / \rho_{r} \rightarrow 0$, in the early-time limit.

Until the effect of curvaton decay becomes significant, the curvaton and radiation densities each satisfy their own energy conservation equation $\dot{\rho}_{i}=-3 H\left(\rho_{i}+P_{i}\right)$. In this regime, each $\zeta_{i}$ is constant on super-horizon 
scales [18]. The evolution of $\zeta$ on these scales is due solely to the change of $f$ in Eq. (19), which yields

$$
\dot{\zeta}=\dot{f}\left(\zeta_{\sigma}-\zeta_{r}\right)=H f(1-f)\left(\zeta_{\sigma}-\zeta_{r}\right)
$$

It is intuitively easy to see how $\zeta$ changes with time as the curvaton density grows relative to the radiation, $\dot{f}>0$. It is straightforward to check that Eq. (21) is consistent with Eq. (8) where the non-adiabatic pressure perturbation is given by

$$
\delta P_{\text {nad }}=\frac{4 \rho_{r} \rho_{\sigma}}{4 \rho_{\sigma}+3 \rho_{r}}\left(\zeta_{\sigma}-\zeta_{r}\right) .
$$

An adiabatic perturbation $\delta \rho_{r} / \dot{\rho}_{r}=\delta \rho_{\sigma} / \dot{\rho}_{\sigma}$ corresponds to the special case

$$
\zeta=\zeta_{r}=\zeta_{\sigma}=\text { constant } .
$$

The curvaton scenario corresponds to the case where the curvature perturbation in the radiation produced at the end of inflation is negligible, $\zeta_{r} \approx 0$. In the approximation of sudden decay [1], $\zeta_{r}$ and $\zeta_{\sigma}$ both remain constant up until decay and the curvature perturbation at decay is therefore

$$
\begin{aligned}
\zeta & \approx f_{\mathrm{dec}} \zeta_{\sigma} \\
& \approx \frac{2}{3} f_{\mathrm{dec}} q\left(\frac{\delta \sigma}{\sigma}\right)_{*},
\end{aligned}
$$

where $f_{\text {dec }}$ is $f$ at the decay epoch, conventionally defined in terms of the decay rate by $H_{\mathrm{dec}}=\Gamma$. We assume that after decay, the pre-existing radiation is either insignificant, or else thermalizes with the decay products with the possible exception of CDM. Since we are assuming that there is only one curvaton field, this is sufficient to ensure that $\zeta$ on cosmological scales will retain the same value until the primordial epoch.

Going beyond the sudden decay approximation, we define a number $r$ by

$$
\begin{aligned}
\zeta & =r \zeta_{\sigma} \\
& =r \frac{1}{3} \frac{\delta \rho_{\sigma}}{\rho_{\sigma}} \\
& =r q \frac{2}{3}\left(\frac{\delta \sigma}{\sigma}\right)_{*},
\end{aligned}
$$

where $\zeta$ is evaluated well after the epoch of curvaton decay and $\zeta_{\sigma}$ is evaluated well before this epoch. In the limit where the curvaton completely dominates the energy density before it decays, $r=1$. In other words, the sudden-decay approximation becomes exact in this limit. The reason is that in this case the curvaton and its decay products constitute a single fluid with a definite $P(\rho)$, so that they have constant curvature perturbation which is equal to $\zeta_{\sigma}$. If the curvaton does not dominate, one has to resort to numerical calculation of the coupled perturbation equations [26], for which one finds

$$
r \approx\left(\frac{\rho_{\sigma}}{\rho}\right)_{\mathrm{dec}} .
$$

The prediction of the curvaton model for the spectrum of the curvature perturbation is

$$
\mathcal{P}_{\zeta}^{\frac{1}{2}}=\frac{2}{3} r \mathcal{P}_{\delta \sigma / \sigma}^{\frac{1}{2}}
$$

with $\mathcal{P}_{\delta \sigma / \sigma}^{\frac{1}{2}}$ given by Eq. (14). The COBE measurement of the CMB quadrupole anisotropy requires [3]

$$
\mathcal{P}_{\zeta}^{\frac{1}{2}}(\mathrm{COBE})=4.8 \times 10^{-5} .
$$

If the curvaton dominates the energy density before it decays $(r=1)$ this implies the following amplitude for the perturbations of the curvaton field

$$
\mathcal{P}_{\delta \sigma / \sigma}^{\frac{1}{2}}=7.2 \times 10^{-5} .
$$

In other words, the typical field (and density) perturbation on cosmological scales must be of order $10^{-4}$ in that case. If the curvaton does not dominate, we need at least $r \gtrsim 10^{-4}$ to get a spectrum of the correct magnitude (since the typical density perturbation can be at most of order 1). We shall now see how the observational bound on non-Gaussianity actually requires a much higher value.

\section{Possible non-Gaussianity of the curvature perturbation}

From Eqs. (15) and (27), the curvature perturbation depends on the curvaton field perturbation through both linear and a quadratic terms. So far we evaluated only the linear contribution, which gives a Gaussian contribution to the curvature perturbation. However, if the quadratic term in the density perturbation of the curvaton field is not negligible, the total curvature perturbation will have a non-gaussian $\left(\chi^{2}\right)$ component.

The relative magnitude of the quadratic term is conventionally specified [5] by a number $f_{\mathrm{NL}}$ (NL meaning 'non-linear'), which nominally determines the nonGaussian contribution to the Bardeen potential according to the formula

$$
\Phi=\Phi_{\text {gauss }}+f_{\mathrm{NL}} \Phi_{\text {gauss }}^{2}
$$

The connection with the Bardeen potential is only nominal, because the relation between $\Phi$ and $\zeta$ is taken [5] to be

$$
\Phi=-\frac{3}{5} \zeta=-\frac{r}{5} \frac{\delta \rho_{\sigma}}{\rho_{\sigma}} .
$$

This relation is actually valid only on super-horizon scales after matter domination, the correct relation at the primordial epoch being more complicated and involving the relative neutrino density [3]. 
Using Eq. (15) we have

$$
\frac{\delta \rho_{\sigma}}{\rho_{\sigma}}=2 \frac{\delta \sigma}{\sigma}+\frac{(\delta \sigma)^{2}}{\sigma^{2}} .
$$

Thus, using Eqs. (33) and (34), the prediction of the curvaton hypothesis is

$$
f_{\mathrm{NL}}=\frac{5}{4 r} .
$$

At this point we have to remember that first-order cosmological perturbation theory is being assumed. Secondorder metric perturbations will also give a correction $\Phi^{(2)}(\mathbf{x})$, presumably with typical magnitude

$$
\left|\Phi^{(2)}(\mathbf{x})\right| \sim \Phi_{\text {gauss }}^{2} .
$$

It follows that the estimate Eq. (36) of $f_{\mathrm{NL}}$ will be valid only in the regime $f_{\mathrm{NL}} \gg 1$ (and that smaller values of $f_{\mathrm{NL}}$ cannot even be defined, unless $\Phi^{(2)}(\mathbf{x})$ is itself the square of a Gaussian quantity). In other words, the validity of the estimate Eq. (36) requires that the curvaton contributes only a small fraction of the energy density just before it decays. In the opposite case that the curvaton dominates the density before it decays, the nonGaussianity calculated according to linear theory is lost in the "noise" of the unknown second-order correction to cosmological perturbation theory.

Now we compare the prediction with present and future data on the CMB anisotropy. A recent analysis of the COBE data 27] yields $\left|f_{\mathrm{NL}}\right| \lesssim 2 \times 10^{3}$, implying the constraint $r \gtrsim 6 \times 10^{-4}$. From Eqs. (30) and (31) we see that this bound is equivalent to $\mathcal{P}_{\delta \sigma / \sigma}^{\frac{1}{2}} \lesssim 0.1$. Starting in 2003, data from the MAP satellite [28] will either detect non-Gaussianity or give $\left|f_{\mathrm{NL}}\right| \lesssim 20$ [5] corresponding to $r \gtrsim 0.06$. If non-Gaussianity is detected by MAP it will clearly be above the noise of the second-order correction. Looking further ahead, the PLANCK satellite will either detect non-Gaussianity or give $\left|f_{\mathrm{NL}}\right| \lesssim 5$ 汭 corresponding to $r \gtrsim 0.2$. Apparent non-Gaussianity at the bottom end of this range would however have to be checked against the second-order order correction to $\Phi$ to make sure that the correction is negligible.

We have seen that the curvaton hypothesis can easily give significant non-Gaussianity. The reason is that the predicted curvature perturbation is proportional to the perturbation of the curvaton density, a quantity which depends on the square of the curvaton field. The perturbation in the curvaton field is supposed to be Gaussian because it is supposed to have negligible interaction, making its Fourier components uncorrelated which is the definition of Gaussianity. But the corresponding perturbation in the curvaton density is a linear combination of the curvaton field perturbation and its square. From a theoretical viewpoint the square could even dominate [29,8], though as we have seen this is ruled out by the data.
This is in sharp contrast with the situation for the inflaton hypothesis, in which the curvature perturbation is purely linear in the inflaton field perturbation. The inflaton can usually be treated as a practically free field, making its perturbations and the curvature practically Gaussian. In particular, for the usual single-field models, where there is an essentially unique inflaton trajectory, the self-interaction of the inflaton field is the only relevant one and is kept small by the flatness conditions $\epsilon, \eta \ll 1$. As a result single-field models give [4] $\left|f_{\mathrm{NL}}\right|=|2 \epsilon-2 \eta| \lesssim 0.1$ which is lost in the noise from the secand-order correction to cosmological perturbation theory. In two-field models, where there is a family of possible inflaton trajectories curved in field space, the inflaton field perturbation at the end of inflation will be a linear combination [30]

$$
\delta \phi_{\text {end }} \propto \cos \theta \delta \phi_{*}+\sin \theta \delta \chi_{*},
$$

where $\phi_{*}$ is the inflaton field at the time of horizon exit and $\chi_{*}$ is the field orthogonal to it. By definition the slope of the potential vanishes in the $\chi$ direction but the higher derivatives might in principle be present corresponding to a self-interaction big enough to generate significant non-Gaussianity. In particular there might be a cubic term $M \chi^{3}$ in the potential [31] (with the unperturbed $\chi_{*}=0$ ) or something more complicated [6]. This setup might generate significant non-Gaussianity, but it obviously requires a special choice of the inflaton trajectory. Even when such a choice is made initially, it might be destabilized by the quantum fluctuation before cosmological scales start to leave the horizon.

We conclude that a detection of non-Gaussianity by MAP would strongly suggest that the primordial curvature perturbation is produced by a curvaton field. Such a detection would imply that the density of the curvaton before it decays reaches no more than $6 \%$ of the total. In the opposite case that the curvaton dominates before it decays, it gives a practically Gaussian curvature perturbation, just as in the inflaton scenario.

\section{ISOCURVATURE PERTURBATIONS}

Thus far we have concentrated upon how the curvaton introduces a large-scale curvature perturbation. Now we consider the isocurvature perturbations that may be imprinted in different particle species after the curvaton decay.

We adopt the 'separate universe' viewpoint, implicit in practically all discussions of perturbations on superhorizon scales. At each epoch, it is assumed that each comoving region with size much bigger than the Hubble

\footnotetext{
${ }^{\ddagger}$ The bound 0.1 comes from the observational bound on the spectral index in the inflaton scenario, $n=2 \eta-6 \epsilon$.
} 
distance looks locally like some unperturbed (RobertsonWalker) universe. If these 'separate universes' are all identical, the cosmological perturbations are said to be adiabatic. Perturbations in matter fields then vanish on slices of uniform energy density, and the curvature perturbation $\zeta$ is the only thing that needs to be specified to determine the evolution of the perturbations after horizon entry.

If the 'separate universes' are not identical there are isocurvature perturbations, so-called because they evolve independently of the curvature perturbation on large scales which can therefore be taken to vanish when considering them. One way of specifying a generic isocurvature perturbation, $\delta x$, is to give its value on uniformdensity slices, related to its value on a different slicing by the gauge-invariant equation

$$
H\left(\frac{\delta x}{\dot{x}}\right)_{\delta \rho=0}=H\left(\frac{\delta x}{\dot{x}}-\frac{\delta \rho}{\dot{\rho}}\right) .
$$

For a set of fluids with energy density $\rho_{i}$, the isocurvature perturbations are instead conventionally defined by the gauge-invariant quantities

$$
\mathcal{S}_{i j}=-3 H\left(\frac{\delta \rho_{i}}{\dot{\rho}_{i}}-\frac{\delta \rho_{j}}{\dot{\rho}_{j}}\right) .
$$

In terms of the individual curvature perturbations defined in Eq. (17) this becomes

$$
\mathcal{S}_{i j}=3\left(\zeta_{i}-\zeta_{j}\right)
$$

For fluids which are 'decoupled' (in the sense that there is no energy transfer) and which have a definite equation of state $P_{i}\left(\rho_{i}\right)$, the $\zeta_{i}$ 's are constant on superhorizon scales [18 and hence so are the isocurvature perturbations (41). In this language, the curvaton density perturbation before it decays corresponds to a constant isocurvature perturbation $S_{\sigma r} \equiv 3\left(\zeta_{\sigma}-\zeta_{r}\right)$ which in the curvaton scenario $\left(\zeta_{r}=0\right)$ reduces to $\mathcal{S}_{\sigma r} \equiv 3 \zeta_{\sigma}$.

At the 'primordial' epoch, before the smallest cosmological scale approaches the horizon, there are four 'decoupled' fluids namely the CDM, baryons, photons and neutrinos (taken to be massless). The conventional definitions Eqs. (2)-(勾) of the three isocurvature perturbations correspond to

$$
\begin{aligned}
\mathcal{S}_{B} & =3\left(\zeta_{B}-\zeta_{\gamma}\right) \\
\mathcal{S}_{\mathrm{cdm}} & =3\left(\zeta_{\mathrm{cdm}}-\zeta_{\gamma}\right) \\
\mathcal{S}_{\nu} & =3\left(\zeta_{\nu}-\zeta_{\gamma}\right),
\end{aligned}
$$

In the standard single-inflaton scenario it is impossible to introduce isocurvature perturbations on large scales at the primordial epoch from the purely adiabatic perturbations existing after the end of inflation [18. But in the curvaton scenario the non-adiabatic nature of the curvaton perturbation after inflation $\left(\mathcal{S}_{\sigma r} \neq 0\right)$ means that it is possible for the curvaton to leave isocurvature perturbations after the curvaton decays even on super-horizon scales, which we term 'residual' isocurvature perturbations.

In addition to the energy densities we shall need to consider 'number' densities $n_{\mathrm{cdm}}, n_{B}, n_{L}$ and $n_{L i}$. They are defined, respectively, as (i) the number density of CDM particles, (ii) the density of baryon number $B$, (iii) the density of lepton number $L$ and (iv) the densities of the three individual lepton numbers $L_{i}(i=\mathrm{e}, \mu$ or $\tau)$. By the 'primordial' era any significant lepton number will be carried entirely by neutrinos, making $n_{L i}$ the difference between neutrino and anti-neutrino number densities. In the unperturbed Universe each number density is proportional to $a^{-3}$ so long as the corresponding quantity is conserved in a comoving volume.

For each of these number densities, it will be useful to consider the curvature perturbations $\tilde{\zeta}_{i}$ on slices of uniform $n_{i}$,

$$
\begin{aligned}
\tilde{\zeta}_{i} & \equiv-\psi-H \frac{\delta n_{i}}{\dot{n}_{i}} \\
& =-\psi+\frac{1}{3} \frac{\delta n_{i}}{n_{i}}
\end{aligned}
$$

In a homogeneous universe the conserved number density $n_{i}$ obeys the evolution equation

$$
\dot{n}_{i}+3 H n_{i}=0 \text {. }
$$

Allowing for large-scale perturbations about the strictly homogeneous background, we obtain the local evolution equation for the first-order perturbation

$$
\dot{\delta n_{i}}+3 H \delta n_{i}-3 n_{i} \dot{\psi}=0
$$

assuming all spatial gradients (e.g., divergence of the particles velocity field) are negligible on large scales. Combining equations 4548 ) we obtain the evolution equation for the curvature perturbation

$$
\dot{\tilde{\zeta}}_{i}=0
$$

Hence we see that $\tilde{\zeta}_{i}$ defined in Eq. 45) remains constant on large-scales so long as the particle number $n_{i}$ is conserved.

Equations (45) and (49) are equivalent to the statement that the perturbation in the fractional number density $\left(\delta n_{i} / n_{i}\right)$ is conserved on flat $(\psi=0)$ spatial hypersurfaces. The perturbations in the number densities are best defined on the flat slicing, because on this slicing the expansion rate with respect to coordinate time is unperturbed [18], leading to the constancy of the fractional perturbations.

This is a significant extension to the case of interacting fluids of the result found in Ref. [18] for the constancy of $\zeta_{i}$ for non-interacting fluids on large-scales. It will prove a powerful tool to describe the possible generation of isocurvature perturbations after curvaton decay in what follows. 


\section{RESIDUAL ISOCURVATURE MATTER PERTURBATIONS}

\section{A. Residual isocurvature CDM perturbation}

Without making any assumption about the nature of the CDM, we take the epoch of its creation as the one after which its particle number $n_{\mathrm{cdm}}$ is conserved. There are several candidates for the CDM particle, such as the axion, the lightest supersymmetric particle (LSP), a stable massive particle (wimpzilla) or primordial black holes. For axions the epoch of creation corresponds to the temperature $\sim 1 \mathrm{GeV}$ at which the effective axion mass rises above the Hubble parameter, and for the conventional LSP it corresponds to the freeze-out temperature roughly of order $10 \mathrm{GeV}$. For very massive particles (wimpzillas) 332,33] or black holes [34,35] the epoch of creation may be the end of slow-roll inflation corresponding to an energy scale $\rho^{\frac{1}{4}}$ up to $10^{16} \mathrm{GeV}$. In the case of wimpzillas it may instead [36] be the epoch of thermal inflation corresponding to perhaps $\rho^{\frac{1}{4}} \sim 10^{6} \mathrm{GeV}$. The CDM might also be created as an out-of-equilibrium decay product of the inflaton, the curvaton or some other particle.

After CDM creation, conservation of the CDM particle number ensures that $\tilde{\zeta}_{\text {cdm }}$ defined by Eq. (45) is constant on super-horizon scales. When the CDM becomes non-relativistic with constant mass, $\tilde{\zeta}_{\text {cdm }}$ reduces to $\zeta_{\mathrm{cdm}}$ defined by Eq. (17). In most of the examples mentioned above, this occurs at the epoch of creation. It could happen though that that CDM created from out-ofequilibrium decay is initially relativistic. Also, the axion mass (induced by the QCD instanton) increases after the epoch of creation, becoming constant only when the temperature falls to $100 \mathrm{MeV}$. In any case, $\tilde{\zeta}_{\text {cdm }}$ will certainly have reduced to $\zeta_{\mathrm{cdm}}$ by the primordial epoch.

To evaluate the primordial isocurvature perturbation $\mathcal{S}_{\text {cdm }}$, we assume that the primordial $\zeta_{\gamma}=\zeta$ corresponding to a zero or small neutrino isocurvature perturbation $\zeta_{\nu}-\zeta_{\gamma}$, postponing until Section $\mathrm{VA}$ the possibility of a significant neutrino isocurvature perturbation. We can then re-write Eq. (43) as

$$
\mathcal{S}_{\mathrm{cdm}}=3\left(\tilde{\zeta}_{\mathrm{cdm}}-\zeta\right) .
$$

As we have mentioned, several different mechanism have been considered for creating the CDM. In most cases, the creation mechanism does not involve any quantity with an isocurvature perturbation, which means that at creation the CDM has no isocurvature perturbation. To put it differently, the 'separate universes' are in these cases identical at the epoch of CDM creation, which means that the number density $n_{\mathrm{cdm}}$ at creation depends only on the local energy density.

The most usual way of obtaining CDM which has an isocurvature perturbation at creation is to suppose that it consists of axions, whose production in each region of space corresponds to the onset of the axion field oscillation in that region. If the axion field has an isocurvature perturbation, a CDM isocurvature perturbation will then be produced at the time of creation. However, the magnitude of the isocurvature CDM perturbation produced in this way is unrelated to that of the curvature perturbation. In other words, there is no reason why CDM originating from an oscillation of the axion field should have a significant isocurvature density at the time of its creation. The same is true for all of the other CDM production mechanisms that have been considered so far.

In what follows we will assume that the CDM number density at the epoch of creation depends only on the local energy density. We expect this to be valid in the absence of any non-adiabatic pressure perturbation, i.e., where the local density also determines the local pressure. The only exception will be the case that the CDM is produced directly by the curvaton decay, which will require separate treatment.

In the curvaton scenario where the CDM is created after the curvaton has decayed (or in the inflaton scenario) the CDM isocurvature perturbation will be zero at the 'primordial' epoch. The basic reason is that the 'separate Universes' in these cases are identical. To proceed more formally, the assumption that there is initially no isocurvature density perturbation means that the CDM number density at creation is uniform $\left(\delta n_{\mathrm{cdm}}=0\right)$ on slices of uniform density (where $\psi=-\zeta$ ). Hence we may evaluate the gauge-invariant expression (45) on a surface of uniform density to obtain $\tilde{\zeta}_{\mathrm{cdm}}=\zeta$, with both sides constant. Going forward to the primordial epoch, when the CDM energy is conserved so that $\zeta_{\text {cdm }}=\tilde{\zeta}_{\text {cdm }}$ we find that the primordial CDM isocurvature perturbation, $\mathcal{S}_{\text {cdm }}$ given by Eq. (43), vanishes.

The situation is dramatically different if the CDM is created before the curvaton decays, or if it is created by the curvaton decay itself. In these cases, the process of curvaton decay creates a 'residual' isocurvature CDM perturbation whose properties are closely tied to the curvature perturbation.

Consider the case that the CDM is created well before the curvaton decays and well before the curvaton comes to dominate the energy density $(f \ll 1$ in Eq. (20)). In this regime the Universe is practically unperturbed and therefore $\tilde{\zeta}_{\text {cdm }}$ is practically zero. At the primordial epoch this gives the residual CDM isocurvature perturbation in Eq. (43) as

$$
\mathcal{S}_{\mathrm{cdm}}=-3 \zeta .
$$

In the notation of Ref. [2] this corresponds to a maximum correlation between curvature and CDM-isocurvature perturbations. The power spectra of the two perturbations have the same spectral index and the isocurvature perturbation has an amplitude three times larger than the adiabatic one. Such a large correlated perturbation is ruled out by current observations which require [2,37] 


$$
\left|\frac{\mathcal{S}_{\text {cdm }}}{\zeta}\right|<1.5 \text { at } 95 \% \text { c.l. . }
$$

Our conclusion is that in the curvaton scenario, $C D M$ cannot be created before the curvaton decays and while the total curvature perturbation remains small, $\zeta \ll r \zeta_{\sigma}$. In particular CDM creation just after inflation ends, such as might occur in the case of wimpzillas or black holes, is incompatible with the curvaton scenario.

If the CDM is created before the curvaton decays, but when the curvaton density has become non-negligible, $f \sim 1$ in Eq. (20), there will be a significant non-adiabatic pressure perturbation, $\delta P_{\text {nad }}$ given by Eq. (22), at the epoch of creation and we can no longer assume that the initial CDM number density is unperturbed on uniform density slices. Instead the actual number density at creation will depend on the mechanism by which the CDM is created, and we leave a detailed investigation of the different cases for future work.

Finally, we consider the case that the CDM is created by the decay of the curvaton itself. The epoch of CDM creation then corresponds to the epoch when the curvaton decay is complete. The resulting local CDM density is then a fixed multiple of the curvaton number density well before decay. The fractional perturbations are thus equal and hence

$$
\tilde{\zeta}_{\mathrm{cdm}}=\zeta_{\sigma} .
$$

Using the definition of $r$ in Eq. (26), this gives at the primordial epoch

$$
\mathcal{S}_{\mathrm{cdm}}=3\left(\frac{1-r}{r}\right) \zeta .
$$

This is negligible if the curvaton comes to dominate before it decays $(r=1)$, the physical reason being that the curvaton perturbation becomes an adiabatic curvature perturbation, so it cannot leave behind any residual isocurvature perturbation. But if the curvaton decays before it dominates, $r \ll 1$, there will be large isocurvature perturbations in the CDM, incompatible with existing observational constraints. Note that in the notation of Ref. [2] the curvature and isocurvature perturbations are anti-correlated, in which case the observational limit becomes

$$
\left|\frac{\mathcal{S}_{\mathrm{cdm}}}{\zeta}\right|<0.2 \text { at } 95 \% \text { c.l. },
$$

much stronger than that for correlated isocurvature perturbations. Observational limits on the amplitude of the anti-correlated isocurvature perturbations thus require the curvaton to effectively dominate, $r>0.9$, if the CDM is created by the curvaton decay.

\section{B. Residual baryon isocurvature perturbation}

Baryon number $B$ may be created directly, in which case we take the epoch of baryogenesis to be the one after which $B$ is conserved. Alternatively $B$ may be created through the conversion of $L$ at the electroweak transition. In the latter case $B-L$ is conserved, and for the present purpose we may take the epoch of baryogenesis as the one when $B-L$ is created. Depending on what type of mechanism operates, the epoch of baryon creation in this sense may be anywhere from the end of inflation to the electroweak transition. To keep the notation simple we take the relevant quantity to be $B$ from now on, with the understanding that $B$ is to be replaced by $B-L$ if that is the relevant quantity.

Until the QCD transition at $T \sim 100 \mathrm{MeV}, B$ represents the difference between the abundances of the typically relativistic particles and anti-particles carrying baryon number, and cannot usefully be associated with an energy density. In this situation we need to use $\tilde{\zeta}_{B}$ the curvature perturbation on slices of uniform $n_{B}$ (given in Eq. (45)). This quantity is constant on large scales at all times after baryon creation, and it reduces to $\zeta_{B}$ (Eq. (17)) after the QCD transition when baryon number is carried by non-relativistic nucleons and nuclei.

The theoretical situation for the baryon isocurvature perturbation is similar to the one for CDM. We make the reasonable assumption that the baryon number density at the epoch of baryogenesis depends only on the local energy density, unless baryogenesis comes the curvaton decay itself. If baryogenesis occurs after the curvaton decays, there will be no primordial baryon isocurvature perturbation. If instead baryon number is generated well before curvaton decay and well before the curvaton dominates, there will be a large residual baryon isocurvature perturbation,

$$
\mathcal{S}_{B}=-3 \zeta .
$$

In contrast with the CDM case, such a perturbation is still marginally compatible with current observational data [2]. The effect on the CMB angular power spectrum of a baryon-isocurvature perturbation is essentially the same as that of a CDM-isocurvature perturbation but the size of the effect is diminished by a factor $\Omega_{B} / \Omega_{\mathrm{cdm}}$ due to the smaller density of baryonic matter [39], so the constraint in Eq. (52) becomes

$$
\left|\frac{\mathcal{S}_{B}}{\zeta}\right|<1.5\left(\frac{\Omega_{\mathrm{cdm}}}{\Omega_{B}}\right) \text { at } 95 \% \text { c.l. . }
$$

An isocurvature perturbation of the form given in Eq. (56) will either be ruled out or observed in the near future, providing in the latter case a smoking gun for the curvaton scenario.

The final possibility is that baryon number is produced by the out-of-equilibrium decay of the curvaton itself, as is the case if we identify the curvaton with the righthanded sneutrino of ref. [38]. Then we have at the primordial epoch, in the sudden decay approximation,

$$
\mathcal{S}_{B}=3\left(\frac{1-r}{r}\right) \zeta .
$$


Current observational limits on the amplitude of such an anti-correlated baryon-isocurvature mode require that the curvaton must dominate the density at decay, $r>0.6$, if the curvaton itself is responsible for baryogenesis.

\section{NEUTRINO ISOCURVATURE PERTURBATIONS}

\section{A. The general formalism}

In this section we discuss the possibility of a neutrino isocurvature density perturbation. As far as we are aware our treatment is the first one taking into account the crucial issue of lepton number.

The era of thermal equilibrium for neutrinos ends just before nucleosynthesis, which almost certainly means that this era determines the abundances of neutrinos and anti-neutrinos at the later 'primordial' epoch. (We are not in this paper considering the possibility that the curvaton decays after nucleosynthesis, and for the present purpose we discount too the possibility that it decays between neutrino decoupling and nucleosynthesis.) Taking that for granted, there is no neutrino isocurvature if the lepton number at decoupling is negligible, because the primordial abundance of the neutrinos is then determined entirely by the photon temperature [39].

We therefore consider the case of non-zero lepton number density $n_{L i}$, with $i=\mathrm{e}, \mu$ or $\tau$. While the neutrinos are effectively massless and in equilibrium, with temperature $T_{\nu}$ this corresponds to occupation numbers

$$
f_{i}(E)=\left[\exp \left(E / T_{\nu} \mp \xi_{i}\right)+1\right]^{-1},
$$

for neutrinos and anti-neutrinos with energy $E$. This gives the following expressions for the total energy density $\rho_{i}$ of neutrinos and anti-neutrinos, and the lepton number density $n_{L i}$ equal to the difference between the number densities of neutrino and anti-neutrinos 40];

$$
\begin{aligned}
\frac{\rho_{i}}{\rho_{\gamma}} & =\frac{7}{8}\left(\frac{T_{\nu}}{T_{\gamma}}\right)^{4} A_{i} \\
\frac{n_{L i}}{n_{\gamma}} & =2.15\left(\frac{T_{\nu}}{T_{\gamma}}\right)^{3} B_{i} .
\end{aligned}
$$

where

$$
\begin{aligned}
& A_{i}=\left[1+\frac{30}{7}\left(\frac{\xi_{i}}{\pi}\right)^{2}+\frac{15}{7}\left(\frac{\xi_{i}}{\pi}\right)^{4}\right], \\
& B_{i}=\left[\left(\frac{\xi_{i}}{\pi}\right)+\left(\frac{\xi_{i}}{\pi}\right)^{3}\right],
\end{aligned}
$$

and

$$
\begin{aligned}
\rho_{\gamma} & =\frac{\pi^{2}}{15} T_{\gamma}^{4}, \\
n_{\gamma} & =\frac{2.40}{\pi^{2}} T_{\gamma}^{3}
\end{aligned}
$$

In the usual case that the lepton asymmetry is negligible, these expressions start to become valid at reheating, and hold with $T_{\nu}=T_{\gamma}$ until positron annihilation, after which

$$
T_{\nu}=(4 / 11)^{\frac{1}{3}} T_{\gamma}
$$

In the case of significant lepton asymmetry that we are considering, there is significant neutrino mixing and the expressions become valid only when $T_{\nu}=T_{\gamma}$ falls to a few $\mathrm{MeV}$. The subsequent evolution is more complicated than in the usual case so that the thermal distribution Eq. (59) is not precisely maintained [40], but following the usual practice we make the approximation that it is maintained so that Eqs. (59)-(66) are all valid after positron annihilation. From now on we take the expressions to refer to that era. The asymmetry parameters $\xi_{i}$ are then constant since the neutrinos are travelling freely leading to $n_{i} \propto T_{\nu}^{3} \propto 1 / a^{3}$.

Big-bang nucleosynthesis (BBN) and large-scale structure (LSS) constrain $\xi_{e}$ and $\left|\xi_{\mu}\right|^{2}+\left|\xi_{\tau}\right|^{2}$. If the favoured large mixing angle (LMA) solution to the solar neutrino problem is correct, neutrino oscillations ensure that the $\xi_{i}$ have a common value $\xi$ 41. In that case, the BBN/LSS constraint is $42-0.01<\xi<0.07$. With this constraint, unperturbed lepton number densities are almost certainly too small to have any observable effect on the CMB anisotropy or large-scale structure (LSS). We shall see, though, that this need not be the case for the residual isocurvature perturbation.

Final confirmation of the LMA is expected in a few months from the Kamland reactor experiment [43], but we shall nevertheless allow independent asymmetry parameters in our analysis. In that case $\mathrm{BBN}$ and $\mathrm{CMB} / \mathrm{LSS}$ together give constraints on $\left|\xi_{\mu}\right|=\left|\xi_{\tau}\right|$ (assumed equal for convenience) and $\xi_{e}$ which are much weaker, namely $\left|\xi_{\mu, \tau}\right| \leq 2.5$ and $\left|\xi_{e}\right| \leq 0.30$ [42]. Note that these limits were obtained assuming a purely adiabatic primordial perturbation spectrum and hence serve only as a rough guide in the case of a correlated curvature and neutrino isocurvature perturbations.

For each individual neutrino species we can calculate the curvature $\zeta_{i}$ on uniform- $\rho_{i}$ hypersurfaces from Eqs. (17) and (60)

$$
\zeta_{i}-\zeta_{\gamma}=\left(\frac{\delta T_{\nu}}{T_{\nu}}-\frac{\delta T_{\gamma}}{T_{\gamma}}\right)+\frac{1}{4} \frac{A_{i}^{\prime}}{A_{i}} \frac{\delta \xi_{i}}{\pi},
$$

and the curvature $\tilde{\zeta}_{i}$ on uniform- $n_{L i}$ hypersurfaces given from Eqs. 45) and (61) as

$$
\tilde{\zeta}_{i}-\zeta_{\gamma}=\left(\frac{\delta T_{\nu}}{T_{\nu}}-\frac{\delta T_{\gamma}}{T_{\gamma}}\right)+\frac{1}{3} \frac{B_{i}^{\prime}}{B_{i}} \frac{\delta \xi_{i}}{\pi},
$$

where $A_{i}^{\prime}=(60 / 7) B_{i}$ and $B_{i}^{\prime}=1+3\left(\xi_{i} / \pi\right)^{2}$. Only when $\delta \xi_{i}=0$ do these two quantities coincide.

As we have noted, a neutrino isocurvature perturbation due to the first term in Eqs. (67) and (68), representing a perturbation in the neutrino to photon temperature ratio, is extremely unlikely since it would have 
to be generated during the extremely short era between neutrino decoupling and nucleosynthesis. We here take the first term to be zero.

Following the path that we trod for the CDM and baryon number perturbations, we define the isocurvature perturbations in the number densities of the three lepton numbers,

$$
\begin{aligned}
\tilde{\mathcal{S}}_{i} & =\frac{\delta n_{L i}}{n_{i}}-\frac{\delta n_{\gamma}}{n_{\gamma}} \\
& =3\left(\tilde{\zeta}_{i}-\zeta_{\gamma}\right) .
\end{aligned}
$$

They determine the perturbations in the asymmetry parameters,

$$
\frac{\delta \xi_{i}}{\pi}=\frac{B_{i}}{B_{i}^{\prime}} \tilde{\mathcal{S}}_{i} \approx \frac{\xi_{i}}{\pi} \tilde{\mathcal{S}}_{i},
$$

where the final equality is valid in the regime $\left(\xi_{i} / \pi\right)^{2} \ll$ 1. These expressions refer to the primordial era, and are valid in the early Universe only back to the epoch of positron annihilation at $T \sim \mathrm{MeV}$. While they are valid, the lepton numbers $L_{i}$ are conserved and the curvature perturbation $\tilde{\zeta}_{i}$ on slices of uniform $n_{L i}$ is constant. At early times though, corresponding to $T$ bigger than a few $\mathrm{MeV}$, neutrino mixing becomes significant and the individual lepton numbers are not defined. Instead there is only the total lepton number density and its perturbation,

$$
\begin{aligned}
n_{L} & =\sum n_{L i} \\
\tilde{\mathcal{S}}_{L} & =\frac{\delta n_{L}}{n_{L}}-\frac{\delta n_{\gamma}}{n_{\gamma}} \\
& =3\left(\tilde{\zeta}_{L}-\zeta_{\gamma}\right) .
\end{aligned}
$$

The total lepton number $L$ is well-defined in the early Universe after the epoch of lepton number creation, which we take to be the one after which $L$ is conserved. While $L$ is conserved, the curvature perturbation $\tilde{\zeta}_{L}$ on slices of uniform $n_{L}$ is constant.

The three primordial quantities $n_{i}$ and their perturbations $\mathcal{S}_{i}$ are not in general determined by $n_{L}$ and $\mathcal{S}_{L}$. They are however determined if the LMA solution to the solar neutrino problem is correct because the three quantities are then equal

$$
\begin{aligned}
n_{L i} & =\frac{1}{3} n_{L} \\
\tilde{\mathcal{S}}_{i} & =\tilde{\mathcal{S}}_{L}
\end{aligned}
$$

If one or more neutrino masses are big enough to give a significant amount of dark matter, it will be necessary to insert the $\delta \xi_{i}$ into the initial occupation number Eq. (59), propagating this initial condition forward with evolution equations. In that case the initial condition requires a knowledge of the three individual isocurvature perturbations $\mathcal{S}_{i}$. Here we make instead the opposite approximation of massless neutrinos. Then, the only required initial condition is the isocurvature perturbation in the total neutrino energy density which is specified by the derived quantity $\mathcal{S}_{\nu}$, defined by Eq. (1). This quantity may be calculated using

$$
\zeta_{\nu}=\frac{\sum \rho_{i} \zeta_{i}}{\sum \rho_{i}},
$$

taking $\rho_{i}$ from Eq. (60) and $\zeta_{i}$ from Eq. (67). The result is

$$
\begin{aligned}
S_{\nu} & =\frac{45}{7} \frac{\sum B_{i}\left(\delta \xi_{i} / \pi\right)}{\sum A_{i}} \\
& =\frac{45}{7} \frac{\sum\left(B_{i}^{2} / B_{i}^{\prime}\right) \tilde{\mathcal{S}}_{i}}{\sum A_{i}} \approx \frac{15}{7} \sum\left(\frac{\xi_{i}}{\pi}\right)^{2} \tilde{\mathcal{S}}_{i},
\end{aligned}
$$

where the final approximation is valid for $\left(\xi_{i} / \pi\right)^{2} \ll 1$.

As far as we are aware, this is the first time that expressions for the neutrino isocurvature perturbation have been given in the most realistic case where it is determined by the (perturbed) lepton asymmetry. The effect of the neutrino isocurvature perturbations has never been tested against observational data while including non-zero chemical potential.

\section{B. Induced matter isocurvature perturbations}

Now we address an issue that is relevant for calculating the amplitude of CDM and baryon isocurvature perturbations in the presence of a neutrino isocurvature perturbation. In the presence of the latter, the equality $\zeta_{\gamma}=\zeta_{\nu}=\zeta$ breaks down, and instead we have

$$
\zeta=\left(1-R_{\nu}\right) \zeta_{\gamma}+R_{\nu} \zeta_{\nu}=\zeta_{\gamma}+\frac{R_{\nu}}{3} \mathcal{S}_{\nu}
$$

where $R_{\nu} \equiv \rho_{\nu} /\left(\rho_{\nu}+\rho_{\gamma}\right)$ is the fraction of the final radiation density in neutrinos, and $R_{\nu} \approx 0.41$ for $\xi_{i} \ll 1$.

The amplitude of the CDM and baryon isocurvature perturbations, calculated assuming $\zeta=\zeta_{\gamma}$, acquire an additional term

$$
\mathcal{S}_{\mathrm{cdm} / B}=3\left(\zeta_{\mathrm{cdm} / B}-\zeta_{\gamma}\right)=3\left(\zeta_{\mathrm{cdm} / B}-\zeta\right)+R_{\nu} \mathcal{S}_{\nu}
$$

Hence for CDM/baryons created after curvaton decay (or in the inflaton scenario), for which $\tilde{\zeta}_{\mathrm{cdm} / B}=\zeta$, we now have a non-zero isocurvature matter perturbation

$$
\mathcal{S}_{\mathrm{cdm} / B}=R_{\nu} \mathcal{S}_{\nu}
$$

for $\mathrm{CDM} /$ baryons created before curvaton decay, with $\tilde{\zeta}_{\mathrm{cdm} / B}=0$, we have

$$
\mathcal{S}_{\mathrm{cdm} / B}=-3 \zeta+R_{\nu} \mathcal{S}_{\nu}
$$

while for CDM/baryons created by curvaton decay, with $\tilde{\zeta}_{\mathrm{cdm} / B}=\zeta_{\sigma}$, we have 


$$
\mathcal{S}_{\mathrm{cdm} / B}=3\left(\frac{1-r}{r}\right) \zeta+R_{\nu} \mathcal{S}_{\nu}
$$

If $S_{\nu} \ll \zeta$, then $\zeta_{\gamma}$ can be identified to high accuracy with $\zeta$, and the changes in $\mathcal{S}_{\mathrm{cdm} / B}$ induced by the neutrino isocurvature perturbations represent a small correction.

These changes do not correspond to any change in the evolution of the CDM or baryons in the early Universe. They would be avoided if we worked instead with the quantities $\hat{\mathcal{S}}_{\mathrm{cdm} / \mathrm{B}}=3\left(\tilde{\zeta}_{\mathrm{cdm} / \mathrm{B}}-\zeta\right)$, that define the perturbations on slices of uniform total density (Eq. (39)). These quantities are independent of $\mathcal{S}_{\nu}$ so that, for example, they always vanish if the CDM or matter is created after curvaton decay with a density depending only on the local energy density. In a similar way, the quantities $\hat{\mathcal{S}}_{i}=3\left(\tilde{\zeta}_{i}-\zeta\right)$ reflect the early Universe situation more directly than the quantities $\tilde{S}_{i}$, as we shall see when discussing leptogenesis from curvaton decay. In all cases though, the unhatted quantities are those commonly used at the 'primordial' era, as a starting point for the forward evolution of the perturbations.

\section{The residual neutrino isocurvature perturbation}

Now we wish to discuss the residual neutrino isocurvature perturbation in the curvaton scenario, along the same lines as for the CDM and baryons. To do this, we assume that the LMA solution is correct so that the primordial lepton number densities have a common value, determined by the total lepton number density that is conserved in the early Universe. Since the lepton asymmetry is small in that case, the neutrino isocurvature perturbation is given to high accuracy by Eq. (79),

$$
\mathcal{S}_{\nu} \approx \frac{45}{7}\left(\frac{\xi}{\pi}\right)^{2} \tilde{\mathcal{S}}_{L}
$$

where the common asymmetry parameter $\xi$ satisfies the nucleosynthesis constraint $|\xi|<0.07$.

To evaluate $\tilde{\mathcal{S}}_{L}$ we can follow closely the previous discussions of CDM and baryon number. We take the epoch of creation of lepton number $L$ to be the one at which this quantity starts to be conserved. We assume that the lepton number isocurvature perturbation at creation is zero or negligible, except in the case that lepton number is created by the curvaton decay. If lepton number is created after curvaton decay, $\tilde{\mathcal{S}}_{L}=0$ and there is no neutrino isocurvature perturbation.

If lepton number is created well before curvaton decay and well before the curvaton dominates the density,

$$
\tilde{\mathcal{S}}_{L}=-3 \zeta_{\gamma}
$$

giving

$$
\mathcal{S}_{\nu}=-\frac{135}{7}\left(\frac{\xi}{\pi}\right)^{2} \zeta_{\gamma}
$$

For $|\xi|<0.07$ and using Eq. (80), we have

$$
\left|S_{\nu}\right|<0.01 \zeta \text {. }
$$

This is very small and may never by observable.

Finally, if lepton number is created by out-ofequilibrium curvaton decay,

$$
\tilde{\mathcal{S}}_{L} \approx 3\left(\frac{1-r}{r}\right) \zeta
$$

If the curvaton does not dominate before it decays, this gives

$$
\begin{aligned}
\mathcal{S}_{\nu} & \approx \frac{135}{7}\left(\frac{1-r}{r}\right)\left(\frac{\xi}{\pi}\right)^{2} \zeta \\
\left|\mathcal{S}_{\nu}\right| & <0.01 \frac{1-r}{r} \zeta \lesssim 15 \zeta,
\end{aligned}
$$

where the final inequality comes from the current bounds on the non-Gaussianity parameter, $f_{\mathrm{NL}}$, given in Eq. (36). This upper bound represents a large anticorrelated neutrino isocurvature component which may already be incompatible with current data, though this possibility is yet to be tested against observations. If MAP further constrains the non-Gaussian parameter, $f_{\mathrm{NL}}$, (rather than detecting non-Gaussianity) the bound will become $\left|\mathcal{S}_{\nu}\right|<0.2 \zeta$.

We note that the neutrino isocurvature velocity mode considered by Bucher et al [39] is hard to generate in a curvaton scenario, since we would expect any velocity perturbation left on large scales after the curvaton decay to be suppressed by factors of order $(k / a H)^{2}$ with respect to the (almost scale-invariant) density perturbation, i.e., generated only by spatial gradients in the curvaton field.

\section{CONCLUSIONS}

In this paper we have reviewed the mechanism by which a curvaton field can generate a large-scale density perturbation after inflation, and gone on to investigate the nature of the primordial perturbation that is produced. In many cosmological models the curvaton scenario can reproduce the purely adiabatic and Gaussian density perturbation (curvature perturbation) that is familiar from the inflaton scenario. In another regime though, it can give large non-Gaussianity, and/or a large 'residual' isocurvature perturbation which is completely correlated with the curvature perturbation and can in principle be present in any or all of the baryonic matter, the CDM and the three neutrino species.

\footnotetext{
${ }^{\S}$ The exact formula is $\hat{\mathcal{S}}_{L}=3 \zeta(1-r) / r$ where $\hat{\mathcal{S}}_{L}=3\left(\tilde{\zeta}_{L}-\zeta\right)$, but the difference is negligible in the regime $(\xi / \pi)^{2} \ll 1$.
} 
The non-Gaussianity of the curvature perturbation $\zeta$ arises if the curvaton fails to dominate the energy density before it decays. The perturbation is described by a $\chi^{2}-$ distribution, whose non-Gaussianity is parameterised by

$$
f_{\mathrm{NL}}=\frac{5}{4 r}
$$

where $r$ is approximately given by the curvaton density just before decay, as a fraction of the total. In 2003, results from MAP satellite 28] will either detect this nonGaussianity or show that the curvaton density is at least a few percent of the total when it decays.

The residual isocurvature perturbation in CDM or baryonic matter arises if the CDM or baryon number is created either before curvaton decay, or by the curvaton decay itself. If it is created significantly before curvaton decay and before the curvaton dominates the energy density, the isocurvature perturbation is given by

$$
S_{\mathrm{cdm}}=-3 \zeta
$$

and similarly for baryons. This large, correlated isocurvature perturbation is ruled out by observation for CDM. In other words, CDM cannot be created significantly before curvaton decay and before the curvaton dominates. This is a strong constraint on the cosmology, implying for example that the curvaton scenario is inconsistent with the CDM creation just after the end of inflation. For baryons the perturbation amplitude is close to current observational limits and will be ruled out or detected in the near future, providing in the latter case a smoking gun for the curvaton scenario.

If the CDM or baryon number is created by the curvaton decay itself,

$$
S_{\mathrm{cdm}}=3\left(\frac{1-r}{r}\right) \zeta,
$$

and similarly for baryons. Unless $r$ is close to 1 , this large anti-correlated contribution is ruled out by observation for both CDM and baryons. In other words, the curvaton decay cannot create CDM or baryons unless the curvaton dominates the energy density before it decays.

For the neutrino isocurvature perturbation we have presented an analysis which, for the first time, includes the crucial issue of lepton number, and is relevant for any cosmology. Although general formulae are given, we focus on the case where the ratio of the neutrino and photon temperature is unperturbed, because the late decoupling of neutrinos makes it very hard to see how it could be otherwise. We also focus on the case that the e, $\mu$ and $\tau$ lepton number densities are equal, which will be ensured by mixing in the early Universe at least if the large mixing angle solution to the solar neutrino problem is correct.

With these assumptions, the isocurvature energy density perturbation $S_{\nu}$ of massless neutrinos is related to the lepton number density isocurvature perturbation $\tilde{\mathcal{S}}_{L}$ by

$$
\mathcal{S}_{\nu}=\frac{45}{7}\left(\frac{\xi}{\pi}\right)^{2} \tilde{\mathcal{S}}_{L}
$$

where $\xi$ is the lepton asymmetry parameter, related to the lepton number density by $\xi=4.02\left(n_{L} / n_{\gamma}\right)$. If the lepton number density is of order the baryon number density as is usually supposed, $|\xi| \sim 10^{-9}$ and the neutrino isocurvature perturbation will be completely undetectable. Observationally though, the lepton density is subject only to the nucleosynthesis bound $|\xi|<0.07$, which may allow a detectable perturbation. However neutrino isocurvature perturbations in the presence of a significant lepton asymmetry have never been tested against observations.

In the curvaton scenario, the formulas for the residual isocurvature perturbation $\tilde{\mathcal{S}}_{L}$ are the same as those for the CDM and baryon perturbations. If leptogenesis occurs after curvaton decay there is no residual neutrino isocurvature perturbation. If it is significantly before curvaton decay and before the curvaton dominates, $\tilde{\mathcal{S}}_{L}=-3 \zeta$ and $\left|\mathcal{S}_{\nu}\right|<0.01 \zeta$ which may never be observable. The interesting result comes in the third case, that the curvaton decay itself causes leptogenesis. Then $\tilde{\mathcal{S}}_{L}=3((1-r) / r) \zeta$, and if the curvaton does not dominate before decay we have a neutrino isocurvature perturbation whose magnitude is related to the non-Gaussianity parameter $f_{\mathrm{NL}}=5 / 4 r$,

$$
\mathcal{S}_{\nu} \approx \frac{135}{7}\left(\frac{\xi}{\pi}\right)^{2}\left(\frac{4}{5} f_{\mathrm{NL}}-1\right) \zeta .
$$

If the present bound on non-Gaussianity, $\left|f_{\mathrm{NL}}\right|<2000$, is saturated this permits a huge effect, presumably already ruled out by observation, and even the expected MAP bound on $\left|f_{\mathrm{NL}}\right|<20$ will still allow a significant effect. Observational bounds on $f_{\mathrm{NL}}$ and $\mathcal{S}_{\nu}$ in this scenario should be obtained jointly, taking into account the correlation of these two quantities with each other and with the curvature perturbation $\zeta$.

In summary, we have shown that different curvaton scenarios offer a number of distinctive observational predictions which may be tested by forthcoming experiments.

Note added: A related paper by Moroi and Takahashi 11 discussing the case of matter isocurvature perturbations in the curvaton scenario appeared while this work was in progress.

\section{ACKNOWLEDGMENTS}

We are grateful to Kostas Dimopoulos, Luca Amendola and Martin Bucher for useful discussions. This work was supported in part by PPARC grants PPA/G/S/1999/00138, PPA/G/S/2000/00115 and PGA/G/O/2000/00466, and by EU grant HPRNCT-2000-00152. DW is supported by the Royal Society. 
[1] R. Trotta, A. Riazuelo and R. Durrer, Phys. Rev. Lett. 87, 231301 (2001) arXiv:astro-ph/0104017.

[2] L. Amendola, C. Gordon, D. Wands and M. Sasaki, Phys. Rev. Lett. 88, 211302 (2002) arXiv:astro-ph/0107089.

[3] A. R. Liddle and D. H. Lyth, "Cosmological inflation and large-scale structure," Cambridge, UK: Univ. Pr. (2000) $400 \mathrm{p}$.

[4] A. Gangui, F. Lucchin, S. Matarrese and S. Mollerach, Astrophys. J. 430, 447 (1994) arXiv:astro-ph/9312033; A. Gangui, Phys. Rev. D 50, 3684 (1994) arXiv:astroph/9406014]; L. M. Wang and M. Kamionkowski, Phys. Rev. D 61, 063504 (2000) arXiv:astro-ph/9907431; A. Gangui and J. Martin, arXiv:astro-ph/9908009.

[5] E. Komatsu and D. N. Spergel, Phys. Rev. D 63 (2001) 063002

[6] D. S. Salopek, Phys. Rev. D 45 (1992) 1139; F. Bernardeau and J. P. Uzan, arXiv:hep-ph/0207295.

[7] N. Bartolo, S. Matarrese and A. Riotto, Phys. Rev. D 65 (2002) 103505 arXiv:hep-ph/0112261.

[8] A. D. Linde and V. Mukhanov, Phys. Rev. D 56, 535 (1997) arXiv:astro-ph/9610219.

[9] D. H. Lyth and D. Wands, Phys. Lett. B 524, 5 (2002) arXiv:hep-ph/0110002.

[10] T. Moroi and T. Takahashi, Phys. Lett. B 522, 215 (2001) arXiv:hep-ph/0110096.

[11] T. Moroi and T. Takahashi, arXiv:hep-ph/0206026.

[12] S. Mollerach, Phys. Rev. D 42, 313 (1990).

[13] K. Enqvist and M. S. Sloth, Nucl. Phys. B 626, 395 (2002) arXiv:hep-ph/0109214.

[14] V. Bozza, M. Gasperini, M. Giovannini and G. Veneziano, arXiv:hep-ph/0206131.

[15] E. J. Copeland, R. Easther and D. Wands, Phys. Rev. D 56, 874 (1997) [arXiv:hep-th/9701082].

[16] J. E. Lidsey, D. Wands and E. J. Copeland, Phys. Rept. 337, 343 (2000) arXiv:hep-th/9909061.

[17] J. M. Bardeen, P. J. Steinhardt and M. S. Turner, Phys. Rev. D 28, 679 (1983).

[18] D. Wands, K. A. Malik, D. H. Lyth and A. R. Liddle, Phys. Rev. D 62, 043527 (2000) arXiv:astro-ph/0003278.

[19] V. F. Mukhanov, H. A. Feldman and R. H. Brandenberger, Phys. Rept. 215, 203 (1992).

[20] J. M. Bardeen, Phys. Rev. D 22, 1882 (1980).

[21] D. H. Lyth, Phys. Rev. D 31, 1792 (1985).

[22] J. Martin and D. J. Schwarz, Phys. Rev. D 57, 3302 (1998) arXiv:gr-qc/9704049.

[23] J. Garcia-Bellido and D. Wands, Phys. Rev. D 53, 5437 (1996) arXiv:astro-ph/9511029

[24] D. H. Lyth, arXiv:hep-ph/0205266.

[25] D. Wands, N. Bartolo, S. Matarrese and A. Riotto, arXiv:astro-ph/0205253.

[26] K. A. Malik, D. Wands and C. Ungarelli, in preparation.

[27] E. Komatsu, B. D. Wandelt, D. N. Spergel, A. J. Banday and K. M. Gorski, Astrophys. J. 566 (2002) 19 arXiv:astro-ph/0107605. M. G. Santos et al., Phys. Rev. Lett. 88 (2002) 241302 [arXiv:astro-ph/0107588].
[28] http://map.gsfc.nasa.gov/

[29] D. H. Lyth, Phys. Rev. D 45 (1992) 3394.

[30] C. Gordon, D. Wands, B. A. Bassett and R. Maartens, Phys. Rev. D 63, 023506 (2001) arXiv:astro-ph/0009131.

[31] N. Bartolo, S. Matarrese and A. Riotto, arXiv:hepph/0112261.

[32] D. H. Lyth, D. Roberts \& M. Smith, Phys. Rev. D 57, 7120 (1998) arXiv:hep-ph/9609441.

[33] D. J. Chung, E. W. Kolb and A. Riotto, Phys. Rev. D 59, 023501 (1999) arXiv:hep-ph/9802238.

[34] L. Randall, M. Soljacic and A. H. Guth, Nucl. Phys. B 472, 377 (1996) arXiv:hep-ph/9512439.

[35] J. Garcia-Bellido, A. D. Linde and D. Wands, Phys. Rev. D 54, 6040 (1996) arXiv:astro-ph/9605094.

[36] L. Hui and E. D. Stewart, Phys. Rev. D 60, 023518 (1999) arXiv:hep-ph/9812345; G. R. Dvali, Phys. Lett. B 459, 489 (1999) arXiv:hep-ph/9905204; M. BasteroGil, E. J. Copeland, J. Gray, A. Lukas and M. Plumacher, arXiv:hep-th/0201040;

[37] L. Amendola, private communication.

[38] K. Hamaguchi, H. Murayama and T. Yanagida, Phys. Rev. D 65, 043512 (2002) arXiv:hep-ph/0109030.

[39] M. Bucher, K. Moodley and N. Turok, Phys. Rev. D 62, 083508 (2000) arXiv:astro-ph/9904231.

[40] A. D. Dolgov, arXiv:hep-ph/0202122; G. G. Raffelt, arXiv:astro-ph/0207220; K. N. Abazajian, J. F. Beacom and N. F. Bell, Phys. Rev. D 66 (2002) 013008 arXiv:astro-ph/0203442; Y. Y. Wong, Phys. Rev. D 66 (2002) 025015 arXiv:hep-ph/0203180.

[41] A. D. Dolgov, S. H. Hansen, S. Pastor, S. T. Petcov, G. G. Raffelt and D. V. Semikoz, Nucl. Phys. B 632 (2002) 363 arXiv:hep-ph/0201287.

[42] M. Orito, T. Kajino, G. J. Mathews and Y. Wang, Phys. Rev. D 65, 123504 (2002) arXiv:astro-ph/0203352.

[43] Talk at Neutrino 2002, for transparancies see http://neutrino2002.ph.tum.de/ 